Carrie A. Jaworski, MD, FAAFP, FACSM; Thomas Moran, MD; Kathryn LaLonde, MS

NorthShore University

Health System Primary Care Sports Medicine, University of Chicago, Pritzker School of Medicine (Drs. Jaworski and Moran); ATC National Athletic Trainers' Association (Ms. LaLonde)

\# cjaworski@northshore. org

The authors reported no potential conflict of interest relevant to this article.

doi: 10.12788/jfp.0109

\title{
At-home exercises for 4 common musculoskeletal complaints
}

\author{
This pictorial review can help you advise patients on how \\ to reduce pain and increase ROM, strength, and balance \\ following acute injury or in chronic impairment.
}

\section{PRACTICE RECOMMENDATIONS \\ > Have patients apply ice} to an acute injury for 15 to 20 minutes at a time to help control inflammation, and prescribe an antiinflammatory medication, if indicated. (A)

> Reserve heat application for use following the acute phase of injury to decrease stiffness. A

> Instruct patients who have an acute lateral ankle sprain to begin "ankle $A B C s$ " and other rangeof-motion exercises once acute pain subsides. (c)

> Consider recommending an eccentric heel stretch to help alleviate plantar fasciitis symptoms. (C)

Strength of recommendation (SOR)

A Good-quality patient-oriented evidence

(B) Inconsistent or limited-quality patient-oriented evidence

C Consensus, usual practice, opinion, disease-oriented evidence, case series setting.
7 he mainstay of treatment for many musculoskeletal (MSK) complaints is physical or occupational therapy. But often an individual's underlying biomechanical issue is one that can be easily addressed with a home exercise plan, and, in light of the COVID-19 pandemic, patients may wish to avoid in-person physical therapy. This article describes the rationale for, and methods of providing, home exercises for several MSK conditions commonly seen in the primary care

\section{General rehabilitation principles:}

First things first

With basic MSK complaints, focus on controlling pain and swelling before undertaking restoration of function. Tailor pharmacologic and nonpharmacologic options to the patient's needs, using first-line modalities such as ice and compression to reduce inflammation, and prescribing scheduled doses of an anti-inflammatory medication to help with both pain and inflammation.

Once pain is sufficiently controlled, have patients begin basic rehabilitation with simple range-of-motion exercises that move the injured region through normal patterns, as tolerated. Later, the patient can progress through more specific exercises to return the injured region to full functional capacity.

Explain to patients that it takes about 7 to 10 days of consistent care to decrease inflammation, but that they should begin prescribed exercises once they are able to tolerate them. Plan a follow-up visit in 2 to 3 weeks to check on the patient's response to prescribed care.

Which is better, ice or heat?

Ice and heat are both commonly used to treat MSK injuries and pain, although scrutiny of the use of either intervention has increased. Despite the widespread use of these modalities, there is little evidence to support their effect on patient 
outcomes. The historical consensus has been that ice decreases pain, inflammation, and edema, while heat can facilitate movement in rehabilitation by improving blood flow and decreasing stiffness. ${ }^{1-3}$ In our practice, we encourage use of both topical modalities as a way to start exercise therapy when pain from the acute injury limits participation. Patients often ask which modality they should use. Ice is generally applied in the acute injury phase (48-72 hours after injury), while heat has been thought to be more beneficial in the chronic stages.

I When and how to apply ice. Applying an ice pack or a bag of frozen vegetables directly to the affected area will help control pain and swelling. Ice should be applied for 15 to 20 minutes at a time, once an hour. If a patient has sensitivity to cold or if the ice pack is a gel-type, have the patient place a layer (eg, towel) between the ice and skin to avoid injury to the skin. Additional caution should be exercised in patients with peripheral vascular disease, cryoglobulinemia, Raynaud disease, or a history of frostbite at the site. ${ }^{4}$

An alternative method we sometimes recommend is ice-cup massage. The patient can fill a small paper cup with water and freeze it. The cup is then used to massage the injured area, providing a more active method of icing whereby the cold can penetrate more quickly. Ice-cup massage should be done for 5 to 10 minutes, 3 to 4 times a day.

I When and how to apply heat. Heat will help relax and loosen muscles and is a preferred treatment for older injuries, chronic pain, muscle tension, and spasms. ${ }^{5}$ Because heat can increase blood flow and, likely, inflammation, it should not be used in the acute injury phase. A heating pad or a warm, wet towel can be applied for up to 20 minutes at a time to help relieve pain and tension. Heat is also beneficial before participating in rehab activities as a method of "warming up" a recently injured area. ${ }^{6}$ However, ice should still be used following activity to prevent any new inflammation.

\section{Anti-inflammatory medications}

For an acute injury, nonsteroidal antiinflammatory drugs (NSAIDs) not only can decrease inflammation and aid in healing but can alleviate pain. We typically start with over-the-counter (OTC) NSAIDs taken on a schedule. A good suggestion is to have the patient take the scheduled NSAID with food for 7 to 10 days or until symptoms subside.

\section{Topical analgesics}

Because oral medications can occasionally cause adverse effects or be contraindicated in some patients, topical analgesics can be a good substitute due to their minimal adverse effects. Acceptable topical medications include NSAIDs, lidocaine, menthol, and arnica. Other than prescribed topical NSAIDs, these products can be applied directly to the painful area on an as-needed basis. Often, a topical patch is a nice option to recommend for use during work or school, and a topical cream or ointment can be used at bedtime.

\section{Graduated rehabilitation}

The following 4 common MSK injuries are ones that can benefit from a graduated approach to rehabilitation at home.

\section{Lateral ankle sprain}

Lateral ankle sprain, usually resulting from an inversion mechanism, is the most common type of acute ankle sprain seen in primary care and sports medicine settings. ${ }^{7-9}$ The injury causes lateral ankle pain and swelling, decreased range of motion and strength, and pain with weight-bearing activities.

Treatment and rehabilitation after this type of injury are critical to restoring normal function and increasing the likelihood of returning to pre-injury levels of activity. ${ }^{9,10}$ Goals for an acute ankle sprain include controlling swelling, regaining full range of motion, increasing muscle strength and power, and improving balance.

I Phase 1: Immediately following injury, have the patient protect the injured area with rest, ice, compression, and elevation (RICE). This will help to decrease swelling and pain. Exercises to regain range of motion, such as stretching and doing ankle "ABCs," should begin within 48 to 72 hours of the initial injury (TABLE 1)..$^{9-11}$

I Phase 2: Once the patient has achieved full range of motion and pain is controlled,
Have patients avoid using heat in the acute injury phase because it can increase inflammation due to increased blood flow. 


\section{TABLE 1}

\section{Rehabilitation exercises for acute ankle sprain} or chronic ankle instability ${ }^{9-11}$

The resistance bands shown below can be found online or at most sporting goods stores.

\section{PATIENT HANDOUT}

\section{Ankle ABCs}

While seated, write out the alphabet in the air with your big toe. Do a set of both upper-and lower-case letters. Your ankle should be moving as you perform this.

\section{Gastrocnemius stretch (ankle dorsiflexion stretch with towel)}

While seated, wrap a towel around the top of your foot and pull so that your toes are pointed toward your head. You should feel a stretch in the back of the leg. Repeat 3 times on each side, holding for 20-30 seconds.

\section{4-way ankle exercise program}

Plantar flexion: Point your toes toward the floor.

While seated, extend your legs in front of you and wrap a resistance band around the foot and hold on to the ends of the band with both hands. Position your foot as shown in each of the 4 photos here. Do all 4 directions. Be sure to keep your heel in contact with the floor the entire time. Perform 10 repetitions.

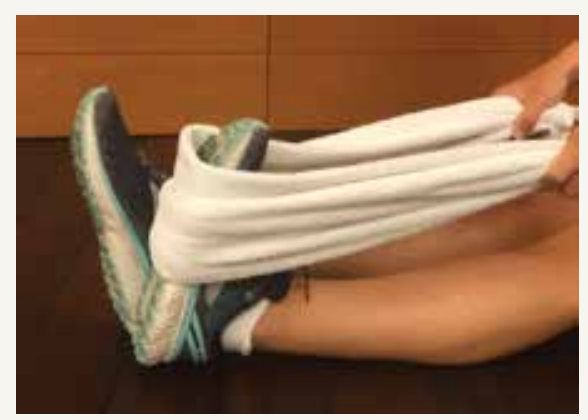

Dorsiflexion: Bring your toes toward your shin.

Perform 10 repetitions.

Eversion: With both legs extended, hold the band with the unaffected foot while you draw your affected foot outward. Perform 10 repetitions.
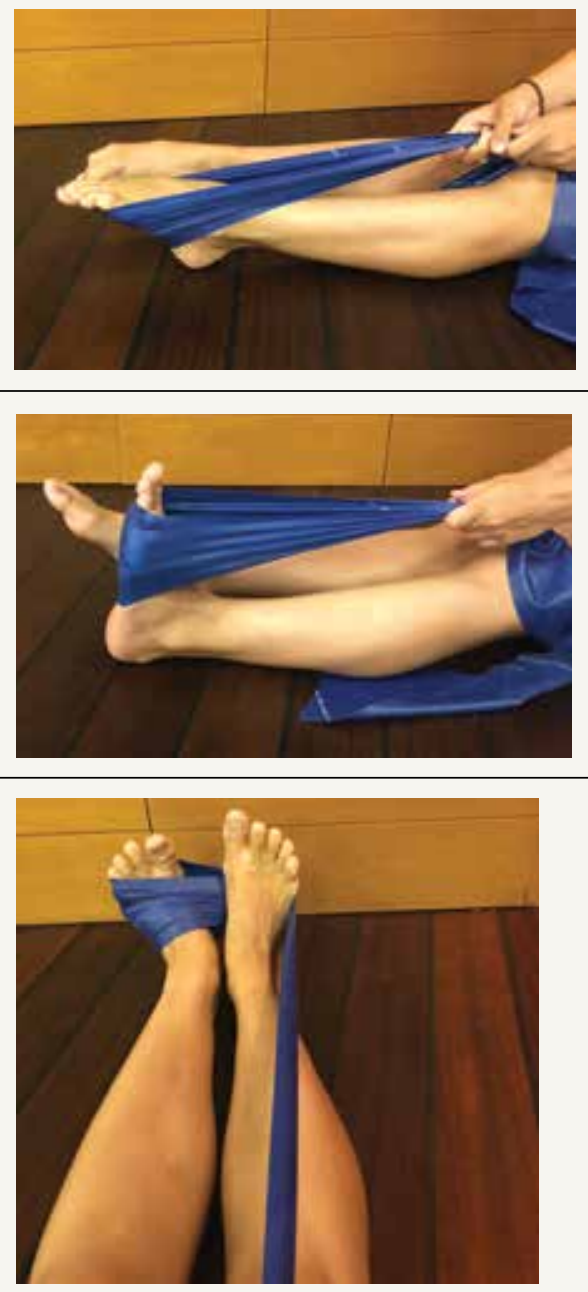
TABLE 1

Rehabilitation exercises for acute ankle sprain or chronic ankle instability ${ }^{9-11}\left(\right.$ cont' $\left.^{\prime} d\right)$

\begin{tabular}{|l|}
\hline \multicolumn{3}{|c|}{ PATIENT HANDOUT } \\
Inversion: Cross the affected leg over the top of the opposite leg \\
and apply pressure to the band while you draw your affected foot \\
inward. Perform 10 repetitions. \\
Single-leg balance \\
Stand and balance on one leg. Perform this next to a table or other \\
sturdy object. Hold your balance in this position. Advanced version: \\
Raise up on your toes while balancing first with your eyes open, \\
then try it with your eyes closed. Hold the position for 30 seconds.
\end{tabular}

begin the process of regaining strength. The 4-way ankle exercise program (with elastic tubing) is an easy at-home exercise that has been shown to improve strength in plantar flexion, dorsiflexion, eversion, and inversion (TABLE 1). . $^{9-11}$

I Phase 3: Once your patient is able to bear full weight with little to no pain, begin a balance program (TABLE $\mathbf{1}^{\text {9-11 }}$ ). This is the most frequently neglected component of rehabilitation and the most common reason patients return with chronic ankle pain or repeat ankle injuries. Deficits in postural stability and balance have been reported in unstable ankles following acute ankle sprains, ${ }^{10,12-15}$ and studies have shown that individuals with poor stability are at a greater risk of injury. ${ }^{13-16}$
For most lateral ankle sprains, patients can expect time to recovery to range from 2 to 8 weeks. Longer recoveries are associated with more severe injuries or those that involve the syndesmosis.

\section{Plantar fasciitis}

Plantar fasciitis (PF) of the foot can be frustrating for a patient due to its chronic nature. Most patients will present with pain in the heel that is aggravated by weight-bearing activities. A conservative management program that focuses on reducing pain and inflammation, reducing tissue stress, and restoring strength and flexibility has been shown to be effective for this type of injury..$^{17,18}$

I Step 1: Reduce pain and inflammation. Deep-tissue massage and cryotherapy 
TABLE 2

Exercises to treat plantar fasciitis ${ }^{17,18}$

\section{PATIENT HANDOUT}

\section{Plantar fascia roll}

Use a frozen water bottle (plastic, no glass). While sitting or standing, roll the bottom of your foot with moderate pressure on top of the bottle. Use as much pressure as you can tolerate without discomfort (for about 5 minutes).

\section{Eccentric heel stretch}

Stand with the balls of your feet on the edge of a stair or curb. Slowly allow both heels to drop down to the point where a stretch is felt in the Achilles tendon. An advanced version is to do this stretch 1 heel at a time. Repeat 3 times on each side, holding the position for $20-30$ seconds.
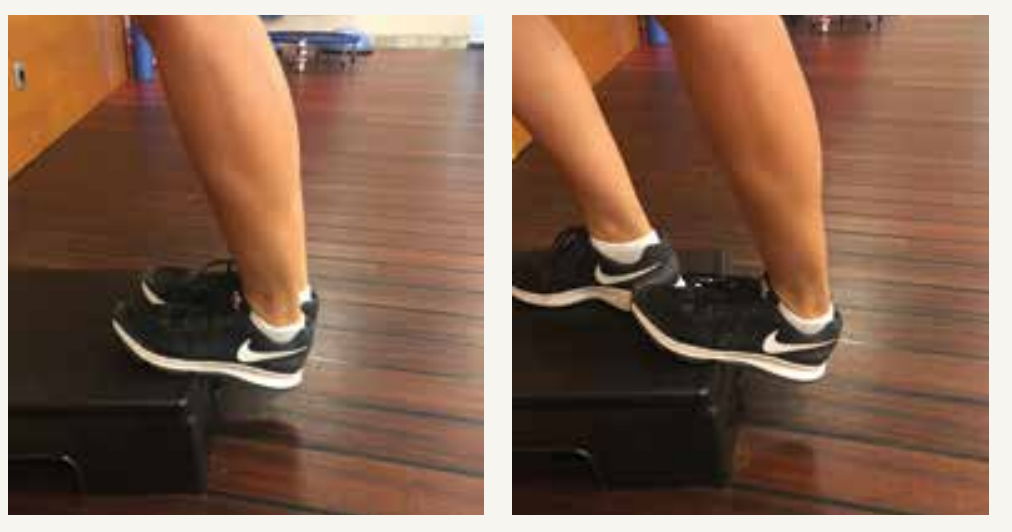

are easy ways to help with pain and inflammation. Deep-tissue massage can be accomplished by rolling the bottom of the foot on a golf or lacrosse ball. A favorite recommendation of ours to reduce inflammation is to use the ice-cup massage, mentioned earlier, for 5 minutes. Or rolling the bottom of the foot on a frozen water bottle will accomplish both tasks at once (TABLE $2^{17,18}$ ).

IStep 2: Reduce tissue stress. Management tools commonly used to reduce tissue stress are OTC orthotics and night splints. The night splint has been shown to improve symptoms, but patients often stop using it due to discomfort. ${ }^{19}$ Many kinds of night splints are available, but we have found that the sock variety with a strap to keep the foot in dorsiflexion is best tolerated, and it should be covered by most care plans.

- Step 3: Restore muscle strength and flexibility. Restoring flexibility of the gastrocnemius and soleus is most frequently recommended for treating PF. Strengthening exercises that involve intrinsic and extrinsic muscles of the foot and ankle are also essential. ${ }^{17,18}$ Helpful exercises include those listed in TABLE 1. ${ }^{9-11}$ Additionally, an eccentric heel stretch can help to alleviate PF symptoms (TABLE $2^{17,18}$ ).

A reasonable timeline for follow-up on newly diagnosed PF is 4 to 6 weeks. While many patients will not have recovered in that time, the goal is to document progress in recovery. If no progress is made, consider other treatment modalities.

\section{Patellofemoral pain syndrome}

Patellofemoral pain syndrome (PFPS) is one of the most common orthopedic complaints, estimated to comprise $7.3 \%$ of all orthopedic visits. ${ }^{20}$ Commonly called "runner's knee," PFPS is the leading cause of anterior knee pain in active individuals. Studies suggest a gender bias, with PFPS being diagnosed more frequently in females than in males, particularly between the ages of 10 and $19 .{ }^{20}$ Often, there is vague anterior knee pain, or pain that worsens with activities such as climbing hills or stairs, or with long sitting or when fatigued.

In general, unbalanced patellar tracking within the trochlear groove likely leads to this pain. Multiple contributory factors have been described; however, evidence increasingly has shown that deficiencies in hip strength may 
TABLE 3

\section{Patellofemoral/hip strengthening exercises ${ }^{24}$}

These exercises should be performed in 3 sets of 10 repetitions on each side.

PATIENT HANDOUT
Son-weightbearing standing hip abduction
from your body while keeping your pelvis level. Repeat on the
opposite side.

contribute significantly to maltracking of the patella with resultant pain. Specifically, weakness in hip external rotators and abductors is associated with abnormal lower extremity mechanics. ${ }^{21}$ One randomized controlled trial by Ferber et al found that therapy protocols directed at hip and core strength showed earlier resolution of pain and greater strength when compared with knee protocols alone. ${ }^{22}$

We routinely talk to patients about how the knee is the "victim" caught between weak hips and/or flat feet. It is prudent to look for both in the office visit. This can be done with one simple maneuver: Ask your patient to do a squat followed by 3 or 4 single-leg squats on each side. This will often reveal dysfunction at the foot/ankle or weakness in the hips/core as demonstrated by pronated feet (along with valgus tracking of the knees inward) or loss of balance upon squatting.

There is general consensus that a nonsurgical approach is the mainstay of treatment for PFPS. ${ }^{23}$ Pelvic stabilization and hip strengthening are standard components along with treatment protocols of exercises tailored to one's individual weaknesses.

Numerous types of exercises do not require specialized equipment and can be taught in the office (TABLE ${ }^{24}$ ). Explain to patients that the recovery process may take 
TABLE 3

\section{Patellofemoral/hip strengthening exercises ${ }^{24}\left(\operatorname{cont}^{\prime} d\right)$}

These exercises should be performed in 3 sets of 10 repetitions on each side.

\section{PATIENT HANDOUT}

\section{Forward lunge}

With your legs spread at shoulder width, lunge forward with the affected lower leg (up to $90^{\circ}$ of flexion) while maintaining a level pelvis and vertical trunk. Be sure to maintain your front knee in line with your ankle. Repeat on unaffected side.

\section{Single-leg squat}

Stand on your affected leg with your hip and knee in $30^{\circ}$ of flexion. Lower your body and then return to the starting position. The goal is to keep your knee aligned over your ankle. Repeat on unaffected side.

several months. Monthly follow-up to document progress is essential and helps to ensure compliance with one's home program.

\section{Neck pain}

The annual prevalence of nonspecific neck pain ranges from $27 \%$ to $48 \%$, with $70 \%$ of individuals being afflicted at some time in their lives. ${ }^{25}$ First rule out any neurologic factors that might suggest cervical disc disease or spinal stenosis. If a patient describes weakness or sensory changes along one or both upper extremities, obtain imaging and consider more formalized therapy with a physical therapist.

In patients without any red flags, investigate possible biomechanical causes. It is es- sential to review the patient's work and home habits, particularly in light of COVID-19, to determine if adjustments may be needed. Factors to consider are desk and computer setups at work or home, reading or laptop use in bed, sleep habits, and frequency of cellular phone calls/texting. ${ }^{26} \mathrm{~A}$ formal ergonomic assessment of the patient's workplace may be helpful.

A mainstay in treating mechanical neck pain is alleviating trapezial tightness or spasm. Manipulative therapies such as osteopathic manipulation, massage, and chiropractic care can provide pain relief in the acute setting as well as help with control of chronic symptoms. ${ }^{27}$ A simple self-care tool is using a tennis ball to massage the trapezial 
TABLE 4

\section{Self-care exercises for acute and chronic neck pain and strains}

\section{PATIENT HANDOUT}

\section{Neck stretches}

Using both hands, apply gentle pressure to the back of your head while allowing your head to flex forward until you feel a stretch along the back of the neck. Repeat this process with one hand applied to the opposite side of your head and gently flex your neck towards that arm. Repeat on the opposite side. Hold each stretch for 10-15 seconds.
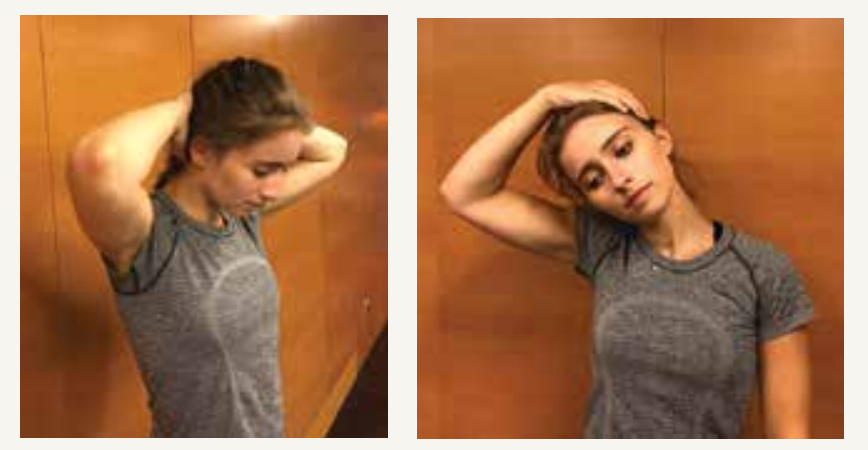

\section{Trapezial release}

Place a tennis ball between you and a wall and lean against the wall. Move up, down, and around to "selfmassage" your neck and shoulders. Another method of self-massage is to put 2 tennis balls in an athletic tube sock and tie off the end. Then place the sock on the floor and lie on it (with your head facing the ceiling). Again, move around to self-massage. Avoid placing the tennis ball directly on your spine.

muscles. This can be accomplished by having the patient position the tennis ball along the upper trapezial muscles, holding it in place by leaning against a wall, and initiating selfmassage. Another method of self-massage is to put 2 tennis balls in an athletic tube sock and tie off the end, place the sock on the floor, and lie on it in the supine position.

There is also evidence that exercise of any kind can help control neck pain. ${ }^{28,29}$ The easiest exercises one can offer a patient with neck stiffness, or even mild cervical strains, is self-directed stretching through gentle pressure applied in all 4 directions on the neck. This technique can be repeated hourly both at work and at home (TABLE 4).

\section{Reminders that can help ensure success}

You can use the approaches described here for numerous other MSK conditions in helping patients on the road to recovery.

After the acute phase, advise patients to

- apply heat to the affected area before exercising. This can help bring blood flow to the region and promote ease of movement.

- continue icing the area following rehabilitation exercises in order to control exercise-induced inflammation.
- report any changing symptoms such as worsening pain, numbness, or weakness.

These techniques are one step in the recovery process. A home program can benefit the patient either alone or in combination with more advanced techniques that are best accomplished under the watchful eye of a physical or occupational therapist. JFP

\section{CORRESPONDENCE}

Carrie A. Jaworski, MD, FAAFP, FACSM, 2180 Pfingsten Road, Suite 3100, Glenview, IL 60026; cjaworski@northshore.org

\section{References}

1. Hubbard TJ, Aronson SL, Denegar CR. Does cryotherapy hasten return to participation? A systematic review. J Athl Train. 2004;39:88-94.

2. Ho SS, Coel MN, Kagawa R, et al. The effects of ice on blood flow and bone metabolism in knees. Am J Sports Med. 1994;22:537-540.

3. Malanga GA, Yan N, Stark J. Mechanisms and efficacy of heat and cold therapies for musculoskeletal injury. Postgrad Med. 2015;127:57-65.

4. Bleakley CM, O'Connor S, Tully MA, et al. The PRICE study (Protection Rest Ice Compression Elevation): design of a randomised controlled trial comparing standard versus cryokinetic ice applications in the management of acute ankle sprain. BMC Musculoskelet Disord. 2007;8:125.

5. Mayer JM, Ralph L, Look M, et al. Treating acute low back pain with continuous low-level heat wrap therapy and/or exercise: a randomized controlled trial. Spine J. 2005;5:395-403.

6. Cetin N, Aytar A, Atalay A, et al. Comparing hot pack, short-wave diathermy, ultrasound, and TENS on isokinetic strength, pain, and functional status of women with osteoarthritic knees: a sin- 
gle-blind, randomized, controlled trial. Am J Phys Med Rehabil. 2008;87:443-451.

7. Waterman BR, Owens BD, Davey S, et al. The epidemiology of ankle sprains in the United States. J Bone Joint Surg Am. 2010;92:2279-2284.

8. Fong DT, Hong Y, Chan LK, et al. A systematic review on ankle injury and ankle sprain in sports. Sports Med. 2007;37:73-94.

9. Kerkhoffs GM, Rowe BH, Assendelft WJ, et al. Immobilisation and functional treatment for acute lateral ankle ligament injuries in adults. Cochrane Database Syst Rev. 2002(3):CD003762.

10. Mattacola CG, Dwyer MK. Rehabilitation of the ankle after acute sprain or chronic instability. J Ath Train. 2002;37:413-429.

11. Hübscher M, Zech A, Pfeifer K, et al. Neuromuscular training for sports injury prevention: a systematic review. Med Sci Sports Exerc. 2010;42:413-421.

12. Emery CA, Meeuwisse WH. The effectiveness of a neuromuscular prevention strategy to reduce injuries in youth soccer: a clusterrandomised controlled trial. Br J Sports Med. 2010;44:555-562.

13. Tiemstra JD. Update on acute ankle sprains. Am Fam Physician. 2012;85:1170-1176

14. Beynnon BD, Murphy DF, Alosa DM. Predictive factors for lateral ankle sprains: a literature review. J Ath Train.2002;37:376-380.

15. Schiftan GS, Ross LA, Hahne AJ. The effectiveness of proprioceptive training in preventing ankle sprains in sporting populations: a systematic review and meta-analysis. J Sci Med Sport. 2015; 18:238-244.

16. Hupperets MD, Verhagen EA, van Mechelen W. Effect of unsupervised home based proprioceptive training on recurrences of ankle sprain: randomised controlled trial. BMJ. 2009;339:b2684

17. Thompson JV, Saini SS, Reb CW, et al. Diagnosis and management of plantar fasciitis. J Am Osteopath Assoc. 2014;114: 900-906.

18. DiGiovanni BF, Nawoczenski DA, Malay DP, et al. Plantar fasciaspecific stretching exercise improves outcomes in patients with chronic plantar fasciitis. A prospective clinical trial with two-year follow-up. J Bone Joint Surg Am. 2006;88:1775-1781.

19. Lee SY, McKeon P, Hertel J. Does the use of orthoses improve self- reported pain and function measures in patients with plantar fasciitis? A meta-analysis. Phys Ther Sport. 2009;10:12-18.

20. Glaviano NR, Key M, Hart JM, et al. Demographic and epidemiological trends in patellofemoral pain. J Sports Phys Ther. 2015;10 281-290.

21. Louden JK. Biomechanics and pathomechanics of the patellofemoral joint. Int J Sports Phys Ther. 2016;11:820-830.

22. Ferber R, Bolgla L, Earl-Boehm JE, et al. Strengthening of hip and core versus knee muscles for the treatment of patellofemoral pain: a multicenter randomized controlled trial. J Ath Train 2015;50:366-377.

23. Collins NJ, Bisset LM, Crossley KM, et al. Efficacy of nonsurgica interventions for anterior knee pain: systematic review and metaanalysis of randomized trials. Sports Med. 2013;41:31-49.

24. Bolgla LA. Hip strength and kinematics in patellofemoral syndrome. In: Brotzman SB, Manske RC eds. Clinical Orthopaedic Rehabilitation. 3rd ed. Philadelphia, PA: Elsevier Mosby; 2011:273-274.

25. Hogg-Johnson S, van der Velde G, Carroll LJ, et al. The burden and determinants of neck pain in the general population: results of the Bone and Joint Decade 2000-2010 Task Force on Neck Pain and Its Associated Disorders. Spine. 2008;33(suppl 4): S39-S51.

26. Larsson B, Søgaard K, Rosendal L. Work related neck-shoulder pain: a review on magnitude, risk factors, biochemical characteristics, clinical picture and preventive interventions. Best Pract Res Clin Rheumatol. 2007;21:447-463.

27. Giles LG, Muller R. Chronic spinal pain: a randomized clinica trial comparing medication, acupuncture, and spinal manipulation. Spine. 2003;28:1490-1502.

28. Bronfort G, Evans R, Anderson A, et al. Spinal manipulation, medication, or home exercise with advice for acute and subacute neck pain: a randomized trial. Ann Intern Med. 2012;156:1-10.

29. Evans R, Bronfort G, Bittell S, et al. A pilot study for a randomized clinical trial assessing chiropractic care, medical care, and self-care education for acute and subacute neck pain patients. IManipulative Physiol Ther. 2003;26:403-411.

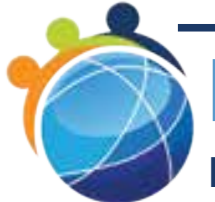

\section{MEDJOBNETWORK $\mathrm{COM}$}

Physician $\bullet$ NP/PA Career Center

\section{The first mobile job board for Physicians, NPs, and PAs}

\section{Mobile Job Searches-access MedJobNetwork.com on the go from your smartphone or tablet}

\section{Advanced Search Capabilities - search for jobs by specialty, job title, geographic location, employer, and more}

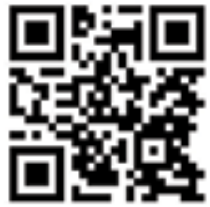

Scan this $Q R$ code to access the mobile version of MedJobNetwork.com

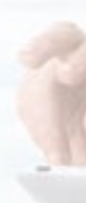

